\title{
DESCRIPCIÓN DEL TAMAÑO DE INCLUSIÓN EN UN ACERO BAJO CARBONO DESOXIDADO CON ALUMINIO MEDIANTE LA DISTRIBUCIÓN GENERALIZADA DE VALORES EXTREMOS*
}

\author{
Edgar Ivan Castro Cedeño ${ }^{1}$ \\ Martin Herrera Trejo ${ }^{2}$ \\ Manuel de Jesus Castro Roman ${ }^{3}$ \\ Monserrat Lopez Cornejo ${ }^{4}$ \\ Fabian Castro Uresti
}

\section{Resumen}

En la producción de aceros limpios el control inclusionario es crítico para la obtención de un producto que cumpla con los requerimientos de calidad. El análisis de la distribución de tamaño de inclusión mediante modelos estadísticos permite estimar la incidencia de inclusiones en el producto solidificado a partir de mediciones tomadas en muestras relativamente pequeñas. En seis coladas de acero bajo carbono desoxidado con aluminio se tomaron muestras de acero a lo largo del procesamiento en horno olla y colada continua. Las muestras se prepararon para estimar mediante análisis metalográfico los tamaños de inclusión. Los datos de tamaño de inclusión se analizaron mediante modelos estadísticos de valores extremos, se hace una comparativa entre la distribución Gumbel (que forma parte de la norma ASTM E-2283) y la distribución Generalizada de Valores Extremos (GEV). Mediante una prueba de hipótesis con el estadístico Anderson-Darling, análisis de cuantiles y con la función de supervivencia se demuestra que la población inclusionaria se describe de una mejor manera con la distribución GEV. Finalmente se da un estimado de la incidencia probable de inclusiones en el producto solidificado.

Palabras-clave: Análisis inclusionario; Estadística de extremos; Control de calidad.

\section{DESCRIPTION OF INCLUSION SIZE FOR A LCAK STEEL WITH THE GENERALIZED EXTREME VALUE DISTRIBUTION}

\begin{abstract}
In the production of clean steels inclusion control is critical for the manufacturing of a product which complies with the required quality specifications. The analysis of the size distribution of inclusions with the use of statistical models allows to estimate the incidence of inclusions in the solidified product from a relatively small metal samples. For six heats of low-carbon aluminum-deoxidized steel, liquid metal samples were taken in the ladle furnace and continuous casting mold. The samples were polished and analyzed by metallographic inspection to determine inclusion sizes in the sample. The measurements of inclusion size were analyzed by means of a statistics of extremes approach in which the Gumbel distribution (which is part of the ASTM E2283 norm) and the Generalized Extreme Value (GEV) distribution are compared with each other. Using the Anderson-Darling hypothesis test, quantile analysis and the survival function it is shown that the estimations made by the GEV model are more representative than the Gumbel model. Finally, an estimate of the probable incidence of inclusions in the solidified product is given.
\end{abstract}

Keywords: Inclusion analysis; Statistics of Extremes; Quality control.

M.C., Metalurgia, Estudiante, Metalurgia, CINVESTAV Saltillo, Ramos Arizpe, Coahuila, México.

PhD, Metalurgia, Profesor, Metalurgia, CINVESTAV Saltillo, Ramos Arizpe, Coahuila, México.

PhD, Metalurgia, Profesor, Metalurgia, CINVESTAV Saltillo, Ramos Arizpe, Coahuila, México.

M.C., Metalurgia, Ing. de proceso, acería, Ternium México, Xoxtla, Puebla, México.

PhD, Metalúrgia, Jefe de Sector, hornos, Ternium México, San Nicolas, Nuevo León, México. 


\section{INTRODUCCIÓN}

Existe una demanda creciente de grados de acero con requerimientos mayores en propiedades mecánicas, de fatiga, resistencia a la corrosión y soldabilidad [1,2]. Esto ha llevado a los aceristas a desarrollar tratamientos del metal líquido complejos con el objetivo de fabricar acero limpio que tenga un nivel de desempeño adecuado. La fabricación de acero limpio se logra mediante el control de la cantidad, tamaño, y composición química de aquellas inclusiones que inevitablemente se presentan en el acero solidificado. Conforme se tiene un acero más limpio, se hace más rara la ocurrencia de inclusiones de gran tamaño, y el tiempo de inspección para encontrarlas mediante metalografía cuantitativa aumenta considerablemente [3].

Una distribución de probabilidad estándar, como la distribución log-normal se puede usar para modelar la distribución de tamaños de una población inclusionaria. Este tipo de distribuciones son un buen modelo para describir la ocurrencia de inclusiones de tamaños cercanos a la moda. Sin embargo, pueden no serlo para inclusiones de gran tamaño que se encuentran en la cola de la distribución, y que se requiera de un modelo complejo para describir el rango completo de mediciones [3]. La teoría estadística de valores extremos describe los tamaños más grandes de un grupo de observaciones mediante un modelo de máximos de bloque que describe la cola de otra distribución mediante un modelo separado, y con una metodología en la que no se necesita analizar una gran superficie para encontrar inclusiones.

\subsection{Descripción del Proceso}

La Figura 1 muestra un esquema de la ruta de producción de la planta de planchón de delgado. Una mezcla de $80 \%$ hierro esponja y $20 \%$ chatarra se carga en el horno de arco eléctrico (EAF) de corriente continua. Después del ajuste químico y de temperatura, el metal líquido se vacía a una olla de 145 toneladas. Durante el vaciado del horno, se agregan de elementos formadores de escoria, ferroaleaciones y cono de aluminio desoxidante directamente al chorro de metal líquido. Así, la desoxidación y desulfuración del metal líquido inician durante la operación de vaciado del horno.

La temperatura y composición química en la olla se homogenizan mediante agitación controlada con argón. Posteriormente la olla se lleva a una estación de horno olla (LF), donde primeramente se acondiciona la escoria para el tratamiento de refinación secundaria. La desoxidación y desulfuración progresan al tiempo que se ajusta la composición química del metal. De ser necesario, se hacen adiciones finas de aluminio mediante inyección de alambre de aluminio.

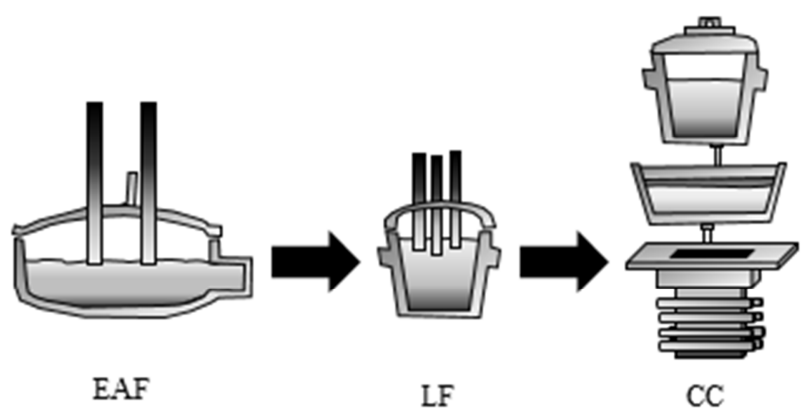

Figura 1. Ruta de procesamiento de la planta de planchón delgado. 


\section{MATERIALES Y MÉTODOS}

\subsection{Pruebas en Planta}

Se estudió la evolución de la población inclusionaria en seis coladas de acero bajo carbono calmado al aluminio (LCAK). Para esto se llevó a cabo un cuidadoso proceso de muestreo de metal en el horno olla (LF) y en el molde de la colada continua (M-CC). En la Tabla 1 se muestra la composición química del acero al final del tratamiento en LF determinada por espectrometría de emisión por chispa. En la Tabla 2 se presenta la posición de muestreo durante el procesamiento y la nomenclatura utilizada para identificar las muestras.

Tabla 1. Análisis químico de las coladas estudiadas

\begin{tabular}{llllllll}
\hline Colada & \%C & \%Mn & \%P & \%S & \%Si & \%Al & \%Ca \\
\hline 1 & 0.0587 & 0.3295 & 0.0057 & 0.0029 & 0.0229 & 0.0309 & 0.0026 \\
\hline 2 & 0.0558 & 0.3545 & 0.0053 & 0.0031 & 0.0299 & 0.0332 & 0.0032 \\
\hline 3 & 0.0643 & 0.4733 & 0.0055 & 0.0029 & 0.0197 & 0.0344 & 0.0037 \\
\hline 4 & 0.0574 & 0.3327 & 0.0030 & 0.0056 & 0.0087 & 0.0300 & 0.0027 \\
\hline 5 & 0.0560 & 0.1534 & 0.0035 & 0.0040 & 0.0167 & 0.0332 & 0.0028 \\
\hline 6 & 0.0458 & 0.1591 & 0.0029 & 0.0021 & 0.0213 & 0.0512 & 0.0027 \\
\hline
\end{tabular}

Tabla 2. Nomenclatura de identificación de muestras y posición de muestreo

\begin{tabular}{ll}
\hline Nomenclatura & Posición de muestreo \\
\hline LF-I & Llegada a LF, después de deoxidation \\
\hline LF-II & Intermedia LF, antes de tratamiento con CaFeAl \\
\hline LF-III & Final LF, después de tratamiento con CaFeAl \\
\hline M-CC & Molde de colada continua, después de colar $100 \mathrm{t}$ \\
\hline
\end{tabular}

\subsection{Análisis Estadístico de Tamaños de Inclusión}

Secciones axiales de las muestras de acero fueron preparadas para un análisis metalográfico convencional mediante desbaste en seco con lijas de carburo de silicio, y pulido con pasta de diamante de 3 y $1 \mu \mathrm{m}$. En cada muestra se analizaron 50 campos a 200 aumentos, que equivale a un área total de medición de 20.062 $\mathrm{mm}^{2}$ por muestra. Mediante análisis de imágenes [4] se estimó el tamaño de la inclusión más grande de cada campo analizado. Para el análisis estadístico se consideró cada colada como una réplica del mismo tratamiento.

En estadística, uno de los estimadores no-paramétricos más simples es la distribución empírica de probabilidad, que está asociada directamente a las mediciones hechas sobre una muestra. La distribución empírica es una función discontinua que ayuda a estimar la función de probabilidad subyacente a la que pertenecen los datos de la muestra. La distribución empírica se define como:

$$
\mathrm{F}_{\mathrm{n}}(x)=\frac{1}{n+1}[\text { Numero de observaciones } \leq x]
$$

donde $\mathrm{n}$ es el número total de observaciones. Una propiedad interesante de la distribución empírica es que se aproxima asintóticamente a uno, es decir, si se tuviera un número infinito de observaciones la probabilidad total sumaría uno.

La aproximación hecha en este trabajo se basa en la rama de la teoría estadística de valores extremos que analiza una serie definida a partir de los máximos de un 
conjunto de bloques, por esta razón se utiliza el tamaño máximo de inclusión medido en cada campo. De acuerdo a la teoría de valores extremos la Distribución Generalizada de Valor Extremo (GEV) es la que se debe utilizar para describir este tipo de series [5]. En la Tabla 3 se presentan las ecuaciones que definen a la Distribución GEV. La variable reducida z es de la misma forma utilizada para la distribución normal clásica, por lo que los parámetros $\sigma$ y $\mu$ son una analogía de la desviación estándar y la media de la muestra. El parámetro de forma k dicta la forma de la cola de la distribución.

Tabla 3. Definición de la Distribución Generalizada de Valores Extremos

\begin{tabular}{|c|c|c|c|}
\hline Parámetros & $\begin{array}{l}\mathrm{k} \text { - parámetro de forma } \\
\sigma \text { - parámetro de escala }(\sigma>0) \\
\mu \text { - parámetro de locación }\end{array}$ & & \\
\hline Variable reducida & $\mathrm{z}=(\mathrm{x}-\mu) / \sigma$ & & \\
\hline \multirow{2}{*}{ Dominio } & $-\infty<x<\infty$ & $\mathrm{k}=0$ & \\
\hline & $1+\mathrm{kz}>0$ & $\mathrm{k} \neq 0$ & \\
\hline \multirow{2}{*}{$\begin{array}{l}\text { Función Densidad } \\
\text { Probabilidad(PDF) }\end{array}$} & $\mathrm{f}(\mathrm{x})=1 / \sigma \exp (-\mathrm{z}-\exp (-\mathrm{z}))$ & $\mathrm{k}=0$ & $(2 a)$ \\
\hline & $\mathrm{f}(\mathrm{x})=1 / \sigma \exp \left(-(1+\mathrm{kz})^{-1 / \mathrm{k}}\right)(1+\mathrm{kz})^{-1-1 / \mathrm{k}}$ & $\mathrm{k} \neq 0$ & $(2 b)$ \\
\hline \multirow{2}{*}{$\begin{array}{l}\text { Función Densidad } \\
\text { acumulada (CDF) }\end{array}$} & $\mathrm{F}(\mathrm{x})=\exp (-\exp (-\mathrm{z}))$ & $\mathrm{k}=0$ & (3a) \\
\hline & $\mathrm{F}(\mathrm{x})=\exp \left(-(1+\mathrm{kz})^{-1 / \mathrm{k}}\right)$ & $k \neq 0$ & (3b) \\
\hline $\begin{array}{l}\text { Función } \\
\text { Supervivencia (S) }\end{array}$ & $S(x)=1-F(x)$ & & (4) \\
\hline
\end{tabular}

En la Figura 2 se ilustra que de acuerdo al valor del parámetro de forma $k$, existen tres casos posibles en la forma de la Distribución GEV. El caso GEV-tipo I, también conocido como distribución Gumbel, tiene parámetro $\mathrm{k}=0$, y describe la cola de distribuciones cuya cola decae exponencialmente. El caso GEV-tipo II tiene parámetro $\mathrm{k}>0$, y describe la cola de distribuciones cuyo cola decae de forma polinómica. El caso GEV-tipo III tiene parámetro de forma $k<0$, y describe la cola de distribuciones cuya cola converge hacia un valor finito. Ekengren y Bergström [6] demostraron que la población inclusionaria puede seguir cualquiera de los tres tipos de la distribución GEV, y que el valor del parámetro de forma k puede cambiar significativamente en un mismo material producido en plantas diferentes con la misma ruta de procesamiento.

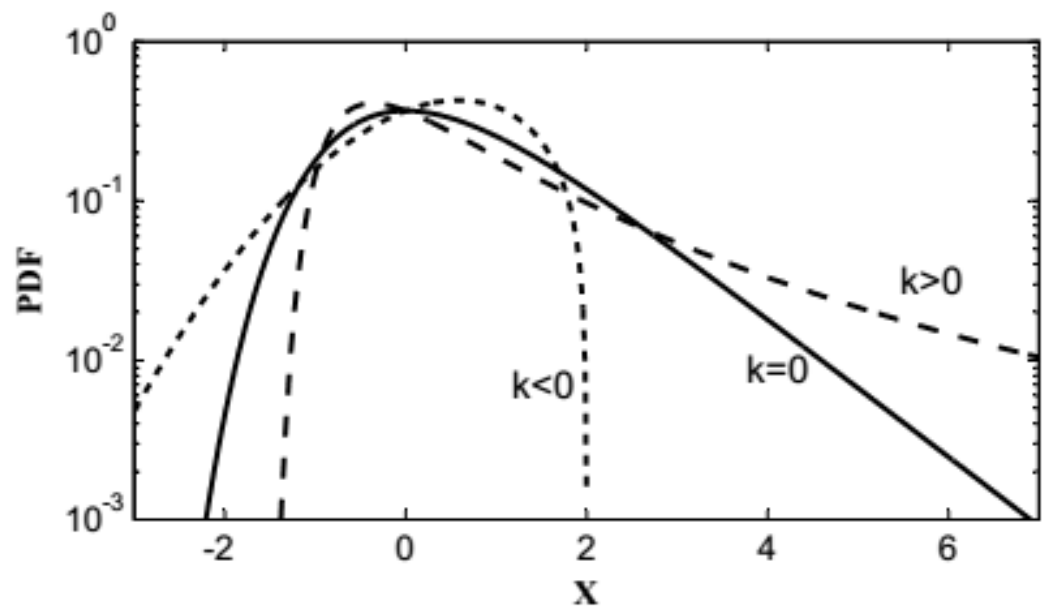

Figura 2. Las tres formas básicas de la distribución GEV. 
Existe un método estandarizado basado en teoría estadística de valores extremos con aplicación práctica para estimar tamaño de inclusiones, es conocido como el método de Estadística de Valores Extremos (SEV). El método SEV estandarizado [79] asume que los datos de tamaño de inclusión máximo por campo se ajustan a la distribución Gumbel, esta suposición debe justificarse antes de utilizar esta distribución para estimar el tamaño de inclusiones [6]. En este trabajo se comparan los resultados de un análisis estadístico utilizando la distribución Gumbel contra el análisis resultante de utilizar la distribución GEV sin asumir previamente el valor del parámetro de forma.

Los parámetros de las distribuciones ajustadas se estimaron con el método de máxima probabilidad (Maximum Likelihood) a partir de los datos de tamaño máximo por campo estimado mediante análisis de imágenes. La probabilidad $L$ está dada por el producto de las PDF para la distribución Gumbel y GEV de las ecuaciones (2a) y (2b) respectivamente. La función de probabilidad L para la distribución es:

$$
\begin{array}{ll}
\mathrm{L}=\prod_{\mathrm{i}=1}^{\mathrm{n}} \frac{1}{\sigma} \exp (-z-\exp (-\mathrm{z})) & \mathrm{k}=0 \\
\mathrm{~L}=\prod_{\mathrm{i}=1}^{\mathrm{n}} \frac{1}{\sigma} \exp \left(-(1+\mathrm{k} z)^{-1 / \mathrm{k}}\right)(1+\mathrm{kz})^{-1-1 / \mathrm{k}} & \mathrm{k} \neq 0
\end{array}
$$

Los estimados de los parámetros $k, \sigma$, y $\mu$ de las distribuciones son aquellos que maximicen el valor de la ecuación (5a) o (5b) dependiendo de si se ajustó la distribución Gumbel o la distribución GEV.

El buen ajuste de las distribuciones ajustadas se comprueba rápidamente mediante el estadístico Anderson-Darling. La prueba Anderson-Darling es una prueba de hipótesis para comprobar si los datos experimentales pertenecen a la distribución ajustada. Esta prueba tiene una mayor sensibilidad a detectar discrepancias sobre la cola de la distribución que sobre la mediana [10]. La ecuación del estadístico Anderson-Darling es:

$\mathrm{A}_{\mathrm{n}}^{2}=-\mathrm{n}-\sum_{\mathrm{i}=1}^{\mathrm{n}} \frac{2 \mathrm{i}-1}{\mathrm{n}} \ln \left(\mathrm{F}\left(\mathrm{x}_{\mathrm{i}}\right)\right)+\ln \left(1-\mathrm{F}\left(\mathrm{x}_{\mathrm{n}+1-\mathrm{i}}\right)\right)$

Donde $x_{1}, \ldots, x_{n}$ son las mediciones experimentales de $n$ observaciones, y $F(x)$ es la CDF de la distribución que se ajustó. La prueba se basa en la hipótesis de que la distribución es de una forma especificada bajo un índice de confianza especificado. La hipótesis se rechaza si el valor del estadístico A es mayor a un valor crítico dependiente de la distribución utilizada y el índice de confianza especificado [11].

\section{RESULTADOS Y DISCUSIÓN}

La Tabla 4 muestra los parámetros de las distribuciones Gumbel y GEV ajustadas a los datos de tamaño máximo de inclusión estimados por análisis de imágenes. En ambas distribuciones los parámetros $\sigma$ y $\mu$ disminuyen al avanzar el procesamiento. La disminución de $\sigma$ es indicativo de la reducción en la dispersión de tamaños de inclusiones, y la disminución $\mu$ es indicativo de una disminución en el tamaño promedio de inclusión En la distribución GEV el valor del parámetro de forma k es positivo en todas las etapas, esto quiere decir que en todo momento la distribución GEV-tipo II es la describe mejor el estado de la población inclusionaria. El valor del parámetro de forma $k$ disminuye conforme se avanza en el procesamiento, esta disminución es indicativo de que el tamaño de la cola izquierda de la distribución disminuye. Como consecuencia la probabilidad de encontrar inclusiones de tamaños grandes, que se localizan sobre la cola izquierda de la distribución, disminuye. 
Igualmente en la Tabla 4 se muestra el valor calculado del estadístico AndersonDarling (A-D) en las distribuciones ajustadas junto con el valor crítico del estadístico para un índice de confianza del 95\%, que es igual a un nivel de significancia ( $p$ value) de 0.05 . Los valores críticos para una significancia de $0.10,0.05$ y 0.01 son $1.933,2.492$ y 3.857 respectivamente [10]. El estadístico indica que la distribución Gumbel no es un buen modelo para describir el estado inclusionario durante el procesamiento en LF porque su valor supera al valor crítico establecido. Para el caso de la distribución GEV en tres de las cuatro etapas el estadístico es menor al valor crítico establecido, indicando que el modelo estadístico GEV describe de mejor manera la población inclusionaria durante el tratamiento en horno olla. La utilización de los valores críticos permite una evaluación rápida del ajuste de las distribuciones.

Tabla 4. Parámetros de las distribuciones estadísticas ajustadas y estadístico A-D

Distribución Gumbel: Distribución GEV:

\begin{tabular}{lccc|cccc}
\hline Etapa & $\boldsymbol{\sigma}$ & $\boldsymbol{\mu}$ & $\mathbf{A - D}$ & $\mathbf{k}$ & $\boldsymbol{\sigma}$ & $\boldsymbol{\mu}$ & $\mathbf{A - D}$ \\
\hline LF-I & 12.802 & 11.008 & 9.5280 & 0.5447 & 7.2958 & 10.147 & 0.8045 \\
\hline LF-II & 6.8710 & 6.0689 & 16.154 & 0.4130 & 4.1167 & 5.7290 & 2.5506 \\
\hline LF-III & 6.6240 & 4.5574 & 8.8182 & 0.3410 & 3.3937 & 5.8502 & 0.7661 \\
\hline M-CC & 4.6737 & 1.8337 & 2.9107 & 0.2189 & 1.6238 & 4.4604 & 0.4909 \\
\hline
\end{tabular}

En la Figura 3 se presenta la evolución de la probabilidad de supervivencia de las inclusiones durante las distintas etapas del procesamiento. La función de supervivencia es el complemento de la CDF, y se interpreta como la probabilidad de encontrar inclusiones mayores a un tamaño determinado. En este estudio el tamaño crítico de inclusión en el producto solidificado es de $20 \mu \mathrm{m}$. Los marcadores redondos representan la función de supervivencia empírica, que resulta de sustituir la ecuación (1) en la ecuación (4). La línea continua representa la función de supervivencia de la distribución Gumbel ajustada, que fue calculada sustituyendo la ecuación (3a) en la ecuación (4). La línea discontinua representa la función de supervivencia de la distribución GEV ajustada, que fue calculada sustituyendo la ecuación (3b) en la ecuación (4). En concordancia con los resultados del estadístico Anderson-Darling, se observa que el modelo de distribución GEV describe de forma adecuada el estado de la población inclusionaria durante el procesamiento en LF, mientras que la distribución Gumbel no lo hace. En la muestra M-CC no se observa discrepancia entre los dos modelos y las mediciones experimentales. Esto es un resultado aparentemente ambiguo, pues bajo la prueba de hipótesis AndersonDarling se determinó que el modelo Gumbel no describe adecuadamente la población inclusionaria en la muestra $\mathrm{M}-\mathrm{CC}$, por lo que requiere de análisis adicional. El desplazamiento hacia la izquierda de los puntos de datos y las líneas de los modelos en la Figura 3 al avanzar el procesamiento es indicativo de que la probabilidad de supervivencia de inclusiones de mayor tamaño disminuye. El cambio entre la muestra LF-III y M-CC se debe a un incremento en la limpieza debido a la flotación de inclusiones durante el tránsito de la olla desde la estación LF a la colada continua y la flotación de inclusiones en el distribuidor de la colada continua. 


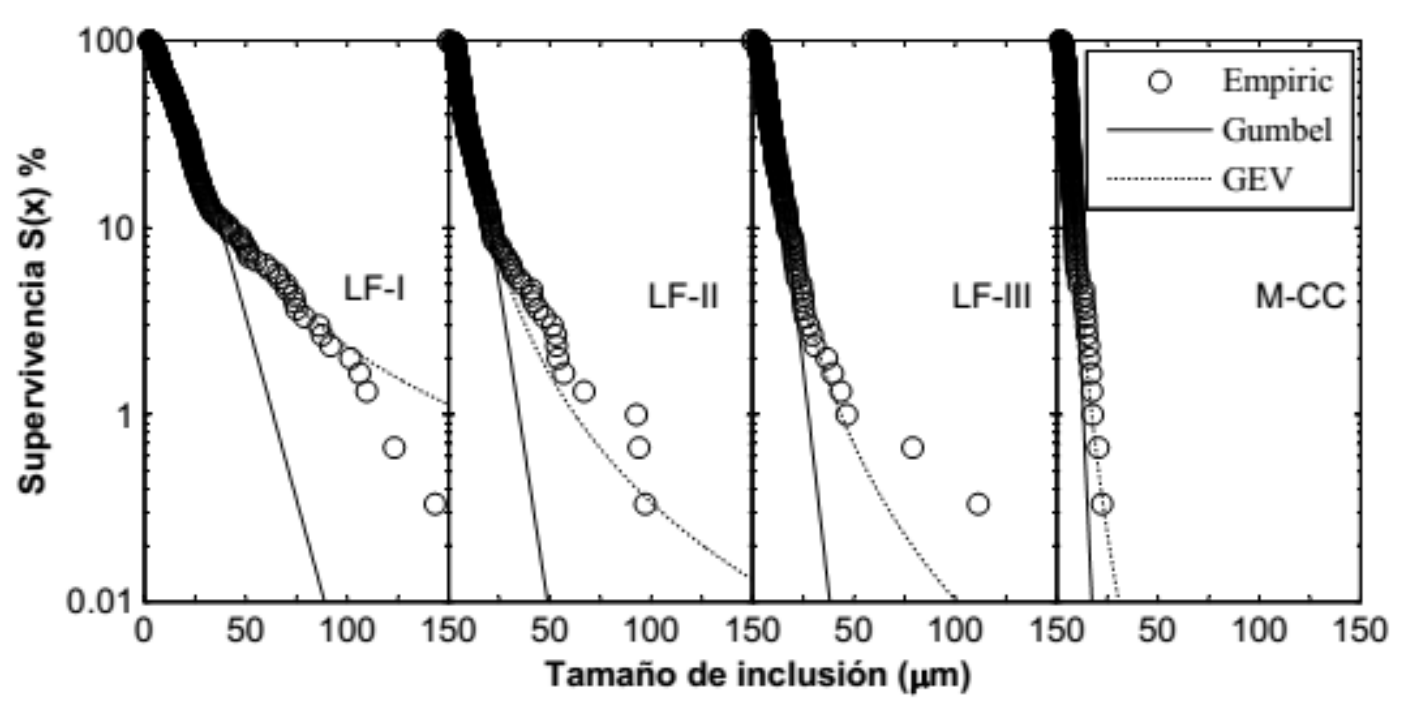

Figura 3. Evolución de la probabilidad de supervivencia de inclusiones durante el procesamiento

En la figura 4 se presenta un gráfico Cuantil-Cuantil, en el que comparan los cuantiles de los valores observados contra los cuantiles de las distribuciones Gumbel y GEV ajustadas para los datos de la muestra M-CC. Los gráficos cuantilcuantil son una de las herramientas gráficas más poderosas para determinar si existen discrepancias en la zona superior de la distribución entre las mediciones experimentales y las distribuciones ajustadas. De acuerdo a la Figura 4 es evidente que el cuantil de $20 \mu \mathrm{m}$ es significativamente menor en la distribución Gumbel que en la muestra, como consecuencia la predicción de sobrevivencia de inclusiones mayores a $20 \mu \mathrm{m}$ no es representativa del estado inclusionario en la muestra M-CC. El gráfico Cuantil-Cuantil de la distribución GEV indica que la predicción de sobrevivencia de inclusiones mayores a $20 \mu \mathrm{m}$ si es representativa del estado inclusionario en la muestra M-CC.

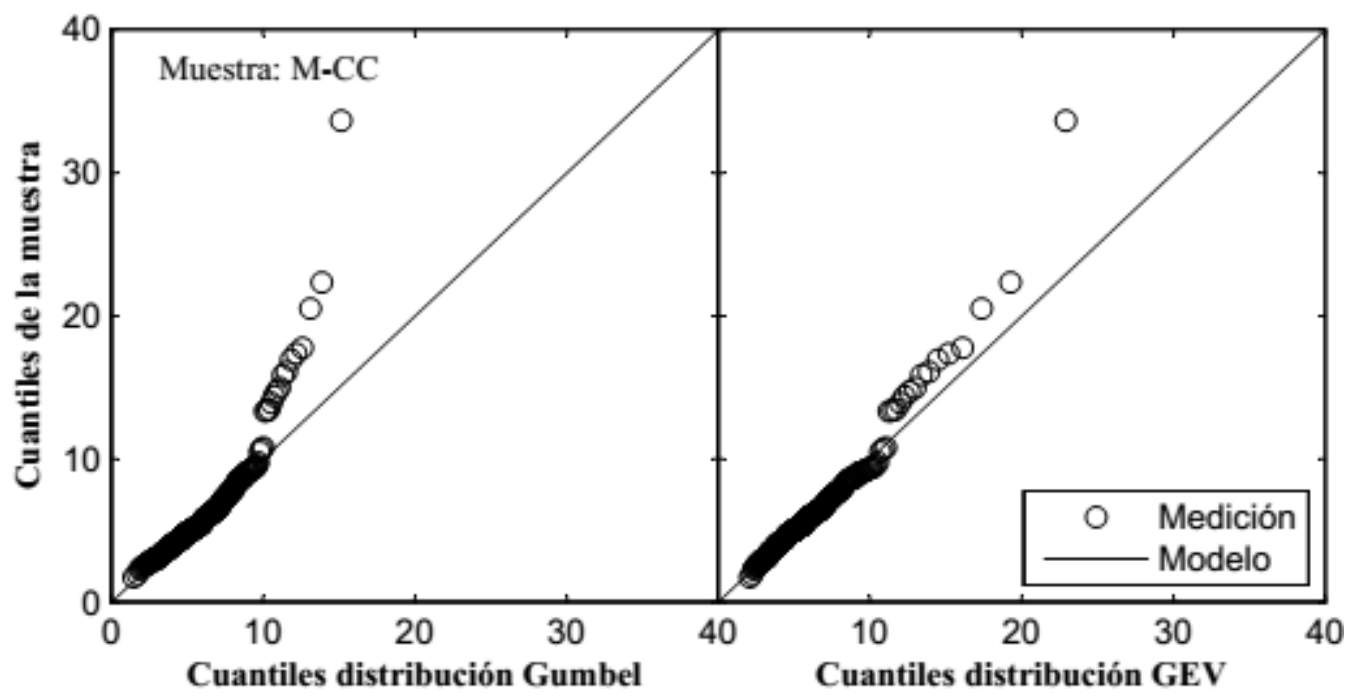

Figura 4. Gráfico cuantil-cuantil para la muestra M-CC

En la Tabla 5, de acuerdo a los resultados del análisis con la distribución GEV, se muestra la probabilidad de supervivencia para inclusiones por encima del tamaño crítico de $20 \mu \mathrm{m}$, el área teórica que debe analizarse para encontrar una inclusión de ese tamaño, y la incidencia esperada en el planchón delgado. La incidencia es el número de inclusiones mayor a un tamaño determinado que de acuerdo al análisis estadístico se podría esperar. La incidencia se calculó considerando que el área 
transversal del planchón delgado producido está en el rango de 49140 a $93296 \mathrm{~mm}^{2}$. El tamaño más extremo de inclusión, cuya incidencia es igual a uno está en el rango de 93.5 a $108 \mu \mathrm{m}$.

Tabla 5. Probabilidad de supervivencia e incidencia esperada de inclusiones en el planchón delgado

\begin{tabular}{|l|l|l|l|}
\hline Tamaño inc. & Supervivencia S(x) $(\mathbf{p p m})$ & Área teórica $\left.\mathbf{( m m}^{\mathbf{2}}\right)$ & incidencia (inc) \\
\hline$>20$ & 5717 & 70 & $700-1330$ \\
\hline$>25$ & 2328 & 172 & $285-541$ \\
\hline$>50$ & 126 & 3187 & $15-29$ \\
\hline$>75$ & 22 & 18646 & $3-5$ \\
\hline$>100$ & 6 & 66452 & 1 \\
\hline
\end{tabular}

\section{CONCLUSIÓN}

Se llevó a cabo un muestreo experimental a fin de evaluar la evolución de la población inclusionaria y determinar la incidencia esperada en el planchón mediante la metodología de estadística de valores extremos. Los resultados permitieron obtener las conclusiones siguientes:

- La metodología de valor extremo se centra en medir las inclusiones de tamaño grande y estimar la probabilidad de que estas ocurran, por lo que puede ser posible llevar a cabo un análisis metalográfico a relativamente pocos aumentos abarcando rápidamente una superficie grande de muestreo.

- El ajuste de cualquier distribución de probabilidad debe ser validado antes de ser considerada como adecuada para llevar a cabo un análisis inclusionario. El estadístico Anderson-Darling permite hacer una validación rápida.

- De acuerdo a las probabilidades de supervivencia dadas por la distribución GEV ajustada, y el tamaño de la sección del planchón solidificado: la incidencia calculada de inclusiones mayores a $20 \mu \mathrm{m}$ es de 700 a 1330 inclusiones, la incidencia de inclusiones mayores a $50 \mu \mathrm{m}$ está en el rango de 15 a 29 inclusiones, y la incidencia de inclusiones mayores a $75 \mu \mathrm{m}$ está en el rango de 3 a 5 inclusiones. El tamaño de inclusión más extremo, que se podría esperar como un evento único está en el rango de 93.5 a $108 \mu \mathrm{m}$.

\section{REFERENCIAS}

1 Lifeng Zhang y B. G. Thomas, State of the Art in Evaluation and Control of Steel Cleanliness, ISIJ International, 2003; 43(3): 271-291.

2 Ahindra Ghosh, Secondary Steelmaking: Principles and Applications, , Boca Ranton, Florida, USA, CRC Press, 2000: 1-7.

3 H. V. Atkinson, G. Shi, Characterization of inclusions in clean steels: a review including the statistics of extremes methods, Progress in Materials Science, 2003; 48(5): 457520.

4 W. S. Rasband, ImageJ y B. M. U. U.S. National Institutes of Health, «ImageJ, Image Processing and Analysis in Java, [acceso el 1504 2015]. Disponible en: http://imagej.nih.gov/ij/

5 R. L. Smith, Statistics of extremes, with applications in environment, insurance and finance, 2001. [acceso el 1504 2015], Disponible en: http://www.stat.unc.edu/postscript/rs/semstatrls.pdf

$6 \mathrm{~J}$. Ekengren y J. Bergström, Extreme value distributions of inclusions in six steels, Extremes, 2012; 15(2): 257-265. 
7 ASTM E-2283-03 Standard Practice for Extreme Value Analysis of Nonmetallic Inclusions in Steel and Other Microstructural Features, ASTM International, 2005.

8 Y. Murakami, Inclusion Rating by Statistics of Extreme Values and Its Application to Fatigue Strength Prediction and Quality Control of Materials, Journal of Research of the National Institute of Standards and Technology, 1994; 99(4): 345-351.

9 Y. Murakami, Metal Fatigue: Effects of Small Defects and Nonmetallic Inclusions, Kidlington, Oxford, UK, Elsevier Science Ltd., 2002: 321-350.

10 T. W. Anderson, D. A. Darling, A test of goodness of fit, Journal of the American Statistical Association, 1954; 49(268): 765-769.

11 National Institute of Standards and Technology, NIST/SEMATECH e-Handbook of Statistical Methods, [acceso el 3004 2014]. [En línea]. Disponible en: http://www.itl.nist.gov/div898/handbook/eda/section3/eda35e.htm 